\title{
FACIAL ATTRACTIVENESS ASSESSMENT USING ILLUSTRATED QUESTIONNAIRERS
}

\author{
ANCA MESAROS ${ }^{1}$, DANIELA CORNEA ${ }^{1}$, LIVIU CIOARA ${ }^{4}$, DIANA DUDEA ${ }^{1}$, \\ MICHAELA MESAROS ${ }^{2}$, MINDRA BADEA ${ }^{3}$
}

\author{
${ }^{1}$ Department of Dental Propaedeutics and Esthetics, Faculty of Dental Medicine, \\ Iuliu Hatieganu University of Medicine and Pharmacy, Cluj-Napoca, Romania \\ ${ }^{2}$ Department of Pediatric Dentistry, Faculty of Dental Medicine, Iuliu Hatieganu \\ University of Medicine and Pharmacy, Cluj-Napoca, Romania \\ ${ }^{3}$ Department of Preventive Dentistry, Faculty of Dental Medicine, Iuliu Hatieganu \\ University of Medicine and Pharmacy, Cluj-Napoca, Romania \\ ${ }^{4}$ Dental practice Turda
}

\section{Abstract}

Introduction. An attractive facial appearance is considered nowadays to be a decisive factor in establishing successful interactions between humans. In relation to this topic, scientific literature states that some of the facial features have more impact then others, and important authors revealed that certain proportions between different anthropometrical landmarks are mandatory for an attractive facial appearance.

Aim. Our study aims to assess if certain facial features count differently in people's opinion while assessing facial attractiveness in correlation with factors such as age, gender, specific training and culture.

Material and methods. A 5-item multiple choice illustrated questionnaire was presented to 236 dental students. The Photoshop CS3 software was used in order to obtain the sets of images for the illustrated questions. The original image was handpicked from the internet by a panel of young dentists from a series of 15 pictures of people considered to have attractive faces. For each of the questions, the images presented were simulating deviations from the ideally symmetric and proportionate face. The sets of images consisted in multiple variations of deviations mixed with the original photo. Junior and sophomore year students from our dental medical school, having different nationalities were required to participate in our questionnaire. Simple descriptive statistics were used to interpret the data.

Results. Assessing the results obtained from the questionnaire it was observed that a majority of students considered as unattractive the overdevelopment of the lower third, while the initial image with perfect symmetry and proportion was considered as the most attractive by only 38.9\% of the subjects. Likewise, regarding the symmetry $36.86 \%$ considered unattractive the canting of the inter-commissural line. The interviewed subjects considered that for a face to be attractive it needs to have harmonious proportions between the different facial elements.

Conclusions. Considering an evaluation of facial attractiveness it is important to keep in mind that such assessment is subjective and influenced by multiple factors, among which the most important are cultural background and specific training.

Keywords: facial aesthetics, image morphing, symmetry, perception, proportion. 


\section{Introduction}

Nowadays, society emphasizes the importance of an attractive physical appearance and especially facial beauty. The face remains a key feature in the determination of human physical attractiveness [1]. Patients are requiring more frequently dental treatments, orthodontic and orthognatic surgical treatments and of course, plasticsurgical treatments for enhancing and optimizing their facial appearance. Social interactions are considered to be influenced by facial looks and certain features are described to play a bigger role than others in interpersonal decisions.

Perception has been defined as the process by which patterns of environmental stimuli are organized and interpreted; it can be influenced by a variety of physical, physiological, and social

factors [2].

Scientific literature includes studies in which facial attractiveness was assessed by showing to panels of judges graphical representations of facial appearances (drawings, silhouettes or photographs) and a rating of such representations was required $[3,4,5,6]$. Some articles present assessments of facial profile attractiveness in cases with antero-posterior skeletal discrepancies [7,8,9], while others assessed the attractiveness of cases with vertical discrepancies [10,11]. Only few studies have inquired upon the attractiveness of cases presenting vertical and symmetry discrepancies $[11,12]$.

Some investigations compared the perception of profile attractiveness between lay people and professionals, others between different categories of clinicians, while many of the studies addressed certain races and ethnic groups $[13,11]$.

A large proportion of the literature emphasizes the importance of the presence of certain relationships such as the golden ratio between different facial proportions as a measure for facial aesthetics in the general public. Ricketts $[14,15]$ was among the first few orthodontists to use the golden ratio to assess the composition of facial hard and soft tissues. Proffit and Fields [16] stated that the vertical height of the mid-face, from the supraorbital ridges to the base of the nose, should equal the height of the lower face, and in the lower face, the mouth should be about one third of the way between the base of the nose and the chin.

High correlations with attractiveness ratings suggest that symmetry along the vertical axis is generally considered a pleasant facial feature $[17,18]$. In addition, the averageness, or prototypicality, of a face has been found to be an important determinant to attractiveness judgments, even when keeping symmetry constant $[19,20,21]$.

Because of the importance shown to the Golden Proportions in assessing facial aesthetics, the relationship between the two needs to be thoroughly investigated.

Manuscript received: 16.09.2014

Accepted: 20.01.2015

Address for correspondence: mesaros.anca@umfcluj.ro
Because there are many factors influencing the perception of beauty such as personal variations, cultural differences, ethnicity, we considered several questions related with perception, such as : how important is specific training in the assessment of attractiveness, are there are differences in perceptions between male and female observers? are there deviations from symmetry or proportion which are perceived by observers as increasing appearance attractiveness, questions to which we wanted to find answers through our study.

\section{Material and methods}

In order to test the hypothesis, a 5-item multiple choice-answer illustrated questionnaire was developed and administered to 236 students from our university.

\section{Illustrated Questionnaire Development}

A panel consisting of five orthodontists was formed in order to search a public image of a beautiful person to be used for the questionnaire. After multiple measurements of symmetry and proportions on several selected photographs, one image of a woman that was found to possess a maximum score in aesthetic measurements was selected (Fig. 1).

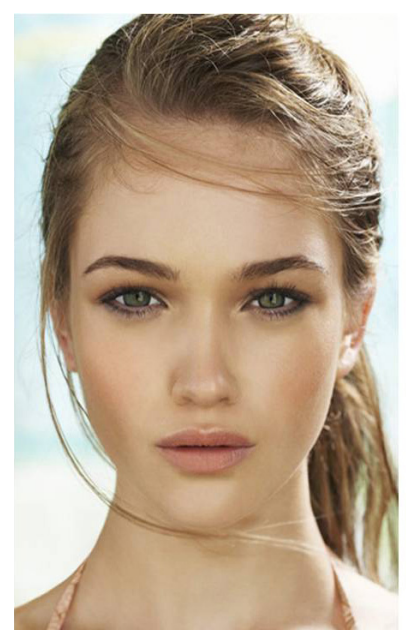

Figure 1. Original image, selected by a pannel of reviewers from the internet: http://renatabeautyandelegance.blogspot.ro/2012/03/ becca-cosmetics.htm

Starting from this selected image, multiple digitally morphed photographs were obtained for the questionnaire. Images were enhanced using the Adobe Photoshop CS 3 software and its extension One Mask pro 4.1 in order to simulate different asymmetries or disproportions from the ideals that it possessed. The obtained images were grouped in three sets containing four and five modified photos together with the original image in order to test the power of discrimination of minor asymmetries and disproportions in the perception of the tested persons.

Images were grouped according to the type of modification that the original photograph suffered as it follows:

The first set contained images where the proportion 
between the vertical facial third was modified: in each of the pictures one of the thirds of the face was increased by $10 \%$ from the original picture.
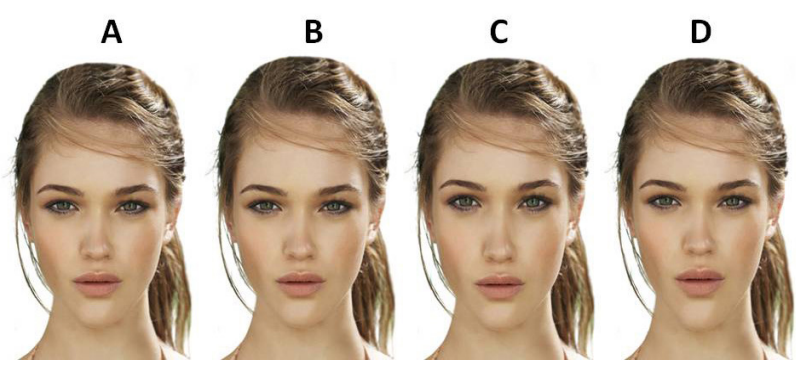

Figure 2. First set of enhanced images: Modifications of facial vertical dimensions: A. Equal vertical thirds of the face, B. Increased upper third, C. Increased middle third, D. Increased lower third.

The second set of images suffered modifications/ deviations of the elements from the mid-saggital line, whereas the third set of images suffered modifications of the horizontal lines, landmarks in symmetry assessment.

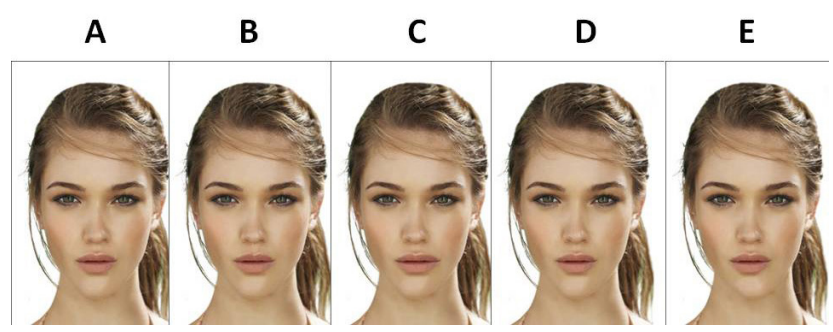

Figure 3. Second set of enhanced images: Modifications from facial symmetry: A. Deviation of the nasal pyramid, B. Deviation of filtrum, C. Deviation of labial angle, D. Image of reference, E. Deviation of menton.
A

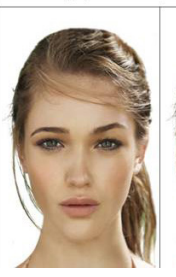

B

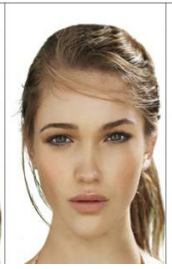

C

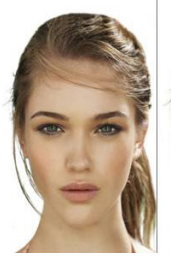

E

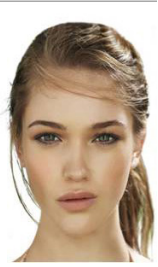

Figure 4. Third set of enhanced images: Deviations of horizontal facial lines: A. Deviation of the eye-brow line, B. Deviation of bipupilar line, C. Image of reference, D. Deviation of subnasal line, E. Deviation of labial line.

To the questions associated with the pictures, two supplemental questions (without connection with the photos) regarding the element of the face which they consider as the most important in facial aesthetics assessment and the conditions that need to be met by an aesthetic face were added.

The resulted questionnaire contained as it follows as it follows:

Personal data information:
Initials

Gender.

Year of study

Q1-3. Which of the following pictures you consider to have the most aesthetic appearance?

Question repeated three times, once for each set of images

Q4. Which element from the face you consider to be the most important in assessing facial aesthetics?
A)The eyes
B)The Nose
C) The Lips
D)The Teeth

Q5. Which of the following conditions you consider to be essential in order to have facial aesthetics?

A) equality between the three vertical thirds of the face;

B) proportionality between facial elements;

C) facial symmetry;

D) harmonious composition between facial elements.

The questionnaire was administered to 236 students from the Faculty of Dentistry of the University of Medicine and Pharmacy Iuliu Hatieganu, Cluj-Napoca, Romania. The questions were projected as a slide-show in an amphitheatre and the subjects chose the answers according to their opinion and marked them on a chart, together with the personal data required. The images were projected without a legend, so the subjects did not know what the difference between images was.

The application of the questionnaire was done in several sequences for students from different years of study. We considered the students from junior years to be inexperienced and with less professional knowledge than students from sophomore years who have been trained in facial aesthetic assessment.

Simple descriptive statistics were used to interpret the data gathered, using Microsoft Excel software, from Microsoft Office 7 package for Windows.

Analyzing the group of subjects we observed that from 236 questioned persons, 111 were males and 125 were women. In regard with their previous experience, 94 subjects were experienced in aesthetic assessment ( students in $5^{\text {th }}$ and $6^{\text {th }}$ year at the Faculty of Dental Medicine), and 142 subjects were considered to be inexperienced (students in $1^{\text {st }}$ and $2^{\text {nd }}$ year at the Faculty of Dental Medicine, Faculty of Dental Technicians).

\section{Results}

Assessing the most pleasant appearance, focused on the first set of images, $38.98 \%$ of the subjects found that an increased upper third of the face was desirable, $29.24 \%$ of subjects appreciated the picture in which all three thirds are equal, as the situation which is the most favorable, $22.46 \%$ opted for an increased middle third while only $9.32 \%$ considered an increased lower third to be more attractive (Fig 5).

Relating to the appraisal of the most pleasant appearance $31.91 \%$ of the experienced group found 
the situation with equality between thirds as the most appreciated, $27.66 \%$ of them appreciated the image where upper third was increased as the situation which is the most favorable, $21.28 \%$ opted for an increased middle third and $19.15 \%$ considered that a lower third increased was more attractive (Fig. 5). On the side of the inexperienced subjects, $46.48 \%$ chose in favor of a dominant upper third, 27.46 percent opted for the equality, $23.2 \%$ chose the increased middle third whereas only 2.82 percent were of the opinion that increased lowest third was more attractive.

During the assessment of the most pleasant appearance, focused on the first set of images, male subjects chose in proportion of $36.04 \%$ the increased upper third followed by an increased middle third, a situation of vertical equilibrium and a least desirable the situation with increased lower third (Fig. 5). Female subjects showed similar preferences.

For the second set of images relating to the assessment of the most pleasant appearance, $26.27 \%$ of the subjects found most pleasing the image of reference, without deviation from symmetry, and the lowest percentage of votes was obtained by the image with a deviated menton $-15.68 \%$. The experienced group had fewer difficulties in finding the image of reference as the most attractive and with fewer votes for the image with a deviated filtrum. The inexperienced group had difficulties in observing differences between images and giving similar percentages for all of the photos.

Based on the second set of images, $22.52 \%$ of male subjects chose the image where a deviation of the corner of the mouth was present, with similar percentages for the deviated filtrum, menton deviation, but disconsidered the image with nasal pyramid deviation $16.22 \%$ (Fig. 6). The group of girls identified in $31.20 \%$ proportion the reference image, without change in the symmetry, $22.40 \%$ preferred the image where the pyramid nasal was diverted, a percentage of $18.40 \%$ opted for the image to the offset filtrum and $12.00 \%$ showed satisfaction with the image in which the menton deflects (Fig. 6).

For the third set of photos, the most positive aspect, $56.36 \%$ of subjects identified the reference image as being the most attractive, $34.32 \%$ appreciated the picture in which the subnasale line is diverted, while the least appreciated was the image with canting of the interlabial line (Fig. 7).

The answers for question 4 identified the most important item in assessing the facial attractiveness of a person: $39.41 \%$ considered the eyes as being the most relevant, $30.08 \%$ of the total sample chose the teeth, $23.31 \%$ were of the opinion that lips are the most important, and $7.20 \%$ of the interviewed have mentioned that the nose is the component which defines facial attractiveness.

From the situations described in question number 5 regarding the primecondition required for facial aesthetics, $36.62 \%$ the subjects found facial symmetry important, $35.21 \%$ opted for facial harmonious composition, $19.72 \%$

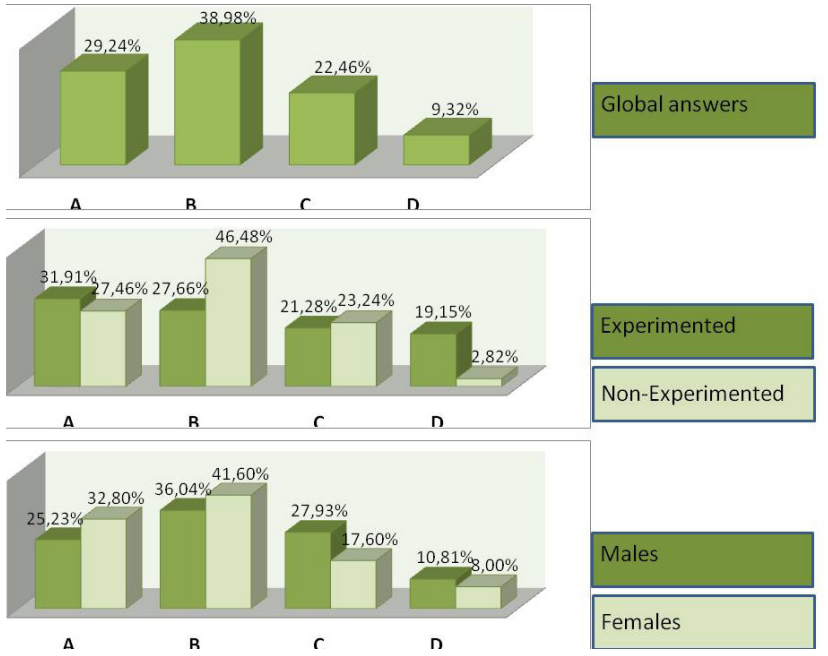

Figure 5. Graphic representation of results obtained for the first question.

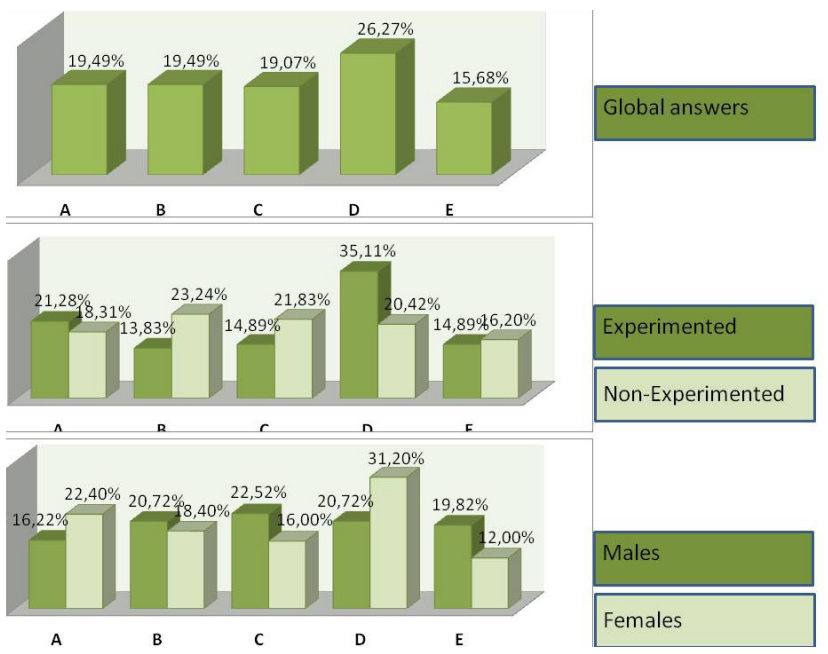

Figure 6. Graphic representation of results obtained related to the second set of images.

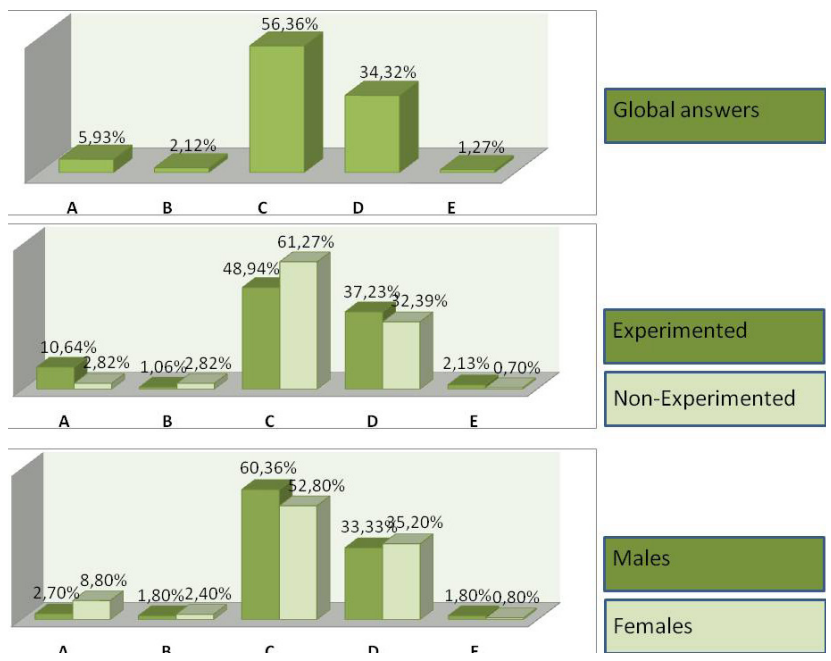

Figure 7. Graphic representation of results obtained for the third question. 
agreed that there should be equality between facial thirds, and $8.45 \%$ considered important the proportionality between elements.

To question number 5, related to the main requirement to be fulfilled when making reference to facial attractiveness, $36.04 \%$ of the male subjects considered facial symmetry important, $34.23 \%$ were in favor of a harmonious composition of facial elements, $17.12 \%$ opted for the equality between facial thirds, and $12.61 \%$ chose to support proportionality between facial elements. For the women, a rate of $52.80 \%$ chose in favor of a harmonious compositions of the elements, $29.60 \%$ considered that the facial symmetry is the most important, $12.00 \%$ opted for equality between facial thirds, and $5.60 \%$ pointed to significant proportionality between facial elements .

\section{Conclusions}

Within the limits of our study, due to a small number of questioned subjects and a low degree of discrimination between images caused by very mild modifications, the following conclusions may be drawn:

1. A dominant upper third of the face is considered to be more attractive than equality between thirds or other disproportion ratios by most people, and only some professionals are able to discriminate small differences-

2. A dominant lower third seems to be the least desirable situation without significant differences between experienced and non experienced evaluators, or between genders.

3. Slight deviations of the elements of the midsagital line are not too visible; therefore even professionals had difficulties in identifying them.

4. The most displeasing deviation from symmetry, between those shown in the second set of image was the deviation of the corner of the mouth.

5. By analyzing closely, the options in this group depended on the degree of experience.For this reason we can state that the perception of aesthetics for male subjects is influenced significantly by the degree of knowledge in this field.

6. The eyes are considered to be the most important element linked to the attractiveness of a person's face, this assertion is proved by the options of all groups concerned

7. The nose seems to have the smallest value in defining facial attractiveness for the questioned subjects.

8. The primary requirement which must be met in relation to facial attractiveness is, in the opinion of a majority, facial symmetry, followed closely by the harmonious composition of the elements of the face. However, the inexperienced population considered in a higher proportion the facial symmetry.

9. Male sex opinions noted in the questionnaire are not much different from those of female sex, but, as said before, the degree of experience in facial aesthetic assessment puts more emphasis on the premium categories.

\section{Aknowledgements}

The present study is part of the Doctoral Thesis entitled "Investigations of the impact of orthodontic treatment upon dento-facial esthetics" elaborated by the first author and was partly financed from the European Social Fund, Project: POSDRU 107/1.5/S/78702 of the Iuliu Hațieganu University of Medicine and Pharmacy , Cluj-Napoca, Romania.

\section{References:}

1. Riggio RE, Widaman KF, Tucker JS, Salinas C. Beauty is more than skin deep: components of attractiveness. Basic and Applied Psychology. 1991;12:423-429.

2. Giddon DB. Orthodontic applications of psychological and perceptual studies of facial esthetics. Semin Orthod. 1995;1:82-93

3. Shaw WC, Rees G, Dawe M, Charles C. The influence of dentofacial appearance on the social attractiveness of young adults. Am J Orthod. 1985;87:21-26.

4. Kenealy P, Frude N, Shaw W. An evaluation of the psychological and social effects of malocclusion: some implications for dental policy making. Soc Sci Med. 1989;28:583-591.

5. Albino NEG, Lawrence DS, Tedesco LA. Psychological and social effects of orthodontic treatment. J Behav Med. 1994;17:8198.

6. Orsini MG, Huang GJ, Kiyak HA, Ramsay DS, Bollen AM, Anderson NK, et al. Methods to evaluate profile preferences for the antero-posterior position of the mandible. Am J Orthod Dentofacial Orthop. 2006;130:283-291.

7. Maganzini AL, Tseng JYK, Epsten J. Perception of facial esthetics by native Chinese participants by using manipulated digital imagery techniques. Angle Orthod. 2000;70:393-399.

8. Kerr WJS, O'Donnell JM. Panel perception of facial attractiveness. Br J Orthod. 1990;24:199-204.

9. Phillips C, Griffin T, Benette E. Perception of facial attractiveness by patients, peers and professionals. Int J Adult Orthodon Orthognath Surg. 1995;10:127-135.

10. Maple JR, Vig KW, Beck FM, Larsen PE, Shanker S. A comparison of providers' and consumers' perception of facial profile attractiveness. Am J Orthod Dentofacial Orthop. 2005;128:690-695.

11. Knight H, Keith O. Ranking of facial attractiveness. Eur J Orthod. 2005;27:340-348.

12. Romani KL, Agahi F, Nanda R, Zernik JH. Evaluation of the horizontal and vertical differences in facial profiles by orthodontists and lay people. Angle Orthod. 1993;63:175-182.

13. De Smit A, Dermaut L. Soft-tissue profile preference. Am J Orthod. 1984;86:67-73.

14. Ricketts RM. The golden divider. J Clin Orthod. 1981;15(11):752-759.

15. Ricketts RM. Golden proportion and Fibonacci series. Am J Orthod. 1982;81(5):351-370.

16. Proffit WR, Fields HW. Contemporary Orthodontics. 3rd ed. St. Louis: Mosby; 1997:157-163.

17. Edler RJ. Background considerations to facial aesthetics. J Orthod. 2001;28(2):159-168.

18. Pancherz H, Knapp V, Erbe C, Heiss AM. Divine proportions in attractive and nonattractive faces. World J Orthod. 2010;11(1):2736.

19. Tatarunaite E, Playle R, Hood K, Shaw W, Richmond S. Facial attractiveness: a longitudinal study. Am J Orthod Dentofacial 


\section{Dental Medicine}

Orthop. 2005;127(6):676-682.

20. Proffit WR, White RP. Surgical- orthodontic treatment. St. Louis: Mosby-Year Book; 1991:105-128.
21. Mills JR. Principles and practice of Orthodontics. Edinburgh: Churchill Livingstone; 1982. 